\title{
Time dependent model bias correction for model based reliability analysis
}

\author{
Zhimin $\mathrm{Xi}^{\mathrm{a}^{*}}$ \\ ${ }^{a}$ University of Tennessee - Knoxville \\ 517 John D. Ticke Building, 851 Neyland \\ Dr., Knoxville, TN 37996-2315 \\ zxi1@utk.edu \\ *Corresponding Author
}

\author{
Hao $\operatorname{Pan}^{\mathrm{b}}$ \\ ${ }^{\mathrm{b}}$ University of Michigan Dearborn \\ 1070 HPEC, 4901 Evergreen Rd, \\ Dearborn, MI 48128 \\ hapan@umich.edu
}

\author{
Ren-Jye Yang ${ }^{\mathrm{c}}$ \\ ${ }^{\mathrm{c}}$ Ford Motor Company \\ 2115 RIC, 2101 Village Rd, \\ Dearborn, MI 48124 \\ ryang@ford.com
}

\begin{abstract}
Model based reliability analysis could be misleading if the simulation model were not validated at the intended design configuration. To improve model accuracy without conceptually revising the model, various model bias correction approaches have been proposed to firstly characterize model bias at training design configurations and then approximate model bias at the intended design configuration. Good accuracy improvement of the model has been shown in literature for not only single model output but also model prediction with multiple or highdimensional outputs. To date, however, the bias correction approaches are mainly proposed for model prediction with time independent (or static) responses and they cannot be directly applied to the model prediction with time dependent responses. This paper presents such a framework of time dependent model bias correction for model based reliability analysis. In particular, three technical components are proposed including: i) an accuracy metric for time dependent model responses under uncertainty, ii) effective approaches for time dependent model bias calibration and approximation, and iii) reliability analysis considering the time dependent model bias. Two case studies including a structural thermal problem and a corroded beam problem are employed to demonstrate the proposed approach for more effective model based reliability analysis.
\end{abstract}

Keywords: Model bias, Reliability analysis, Accuracy metric, Bias correction, Bias calibration and approximation

\section{Introduction}

To date, reliability analysis relies significantly on computer simulation models or analytical models to predict the performance of interest given a set of design configurations. Majority of current research focuses on the development of various reliability analysis methods so that reliability evaluation can be conducted more accurately and efficiently. However, it is well known that models are built to approximate real physical systems on the basis of a series of assumptions and simplifications. Hence, model bias, i.e., the inherent model inadequacy for representing the real system, always exists because there is no perfect model that can represent the real physical system without any error. Ignorance of the model bias in reliability analysis or reliability based design could result in significant design errors by overestimating the system or structure reliability.

The key objective in model validation is to determine the degree to which the model is an accurate representation of the real world from the perspective of the intended uses of the model [1-3]. Traditionally, the research on validation of a simulation/analytical model was proposed to revise the model conceptually for credibility improvement of the model. From the model development perspective, the key advantage of revising the model conceptually is that accuracy of the model could be significantly improved. However, this approach is practically difficult and yet may not be feasible in reality due to three reasons: i) identification of the root cause for model inaccuracy is complicate particularly for large scale engineering systems; ii) fundamental modification of the model is time consuming, costly, and yet may not be practical; and iii) there is no perfect model that can represent the real physical system without any model bias.

The bias correction approach to quantify the model bias in the design domain, therefore, has recently gained significant attention [4-7]. The essential idea is to add the characterized model bias to the baseline simulation/analytical model so that the corrected model prediction could be more accurate and robust compared to 
the baseline model. This so called bias correction approach is mainly composed of three steps: i) characterization of model bias at a few training design configurations, ii) construction of a response surface for the model bias, and iii) approximation of model bias at the intended uses of the model and add it back to the baseline model prediction. The key research challenge is how to approximate model bias effectively based on available training data sets. Otherwise, adding the model bias to the baseline model could worsen the model accuracy [8].

To date, majority of the research in this field focuses on three topics: i) development of model accuracy metric considering limited test data, ii) correct characterization of the model uncertainty (i.e., uncertainty of the model bias) considering test uncertainty and model parameter uncertainty at available design configurations, and iii) development of various models for model uncertainty approximation. Good accuracy improvement of the model has been shown in literature for not only single model output but also model prediction with multiple or highdimensional outputs [4-9]. However, it is worth noting that the proposed approaches are only applicable for model prediction with time independent responses. In other words, model uncertainty is characterized either by a univariate distribution or by a constant value at a given design configuration. New research challenges have to be addressed when model responses are time dependent such as: i) how to characterize model uncertainty as a random process considering limited test data and model parameter uncertainty; ii) how to build response surface for model uncertainty with the form of a random process; iii) how to extend the model accuracy metric for time dependent responses; and iv) how to effectively consider time dependent model bias for reliability analysis and design. Resolving these challenges is imperative because accurate prediction of time dependent responses is critical in many applications especially for time dependent reliability analysis. The contribution of this paper is to generalize the bias correction approach from time independent to time dependent model responses by addressing aforementioned challenges and further enable its seamless integration with advanced probability analysis methods for reliability analysis.

The rest of the paper is organized as follows. Section 2 provides a brief literature review of the bias correction approach. Section 3 elaborates the proposed framework and detailed technical approaches for addressing aforementioned research challenges. Section 4 presents two engineering case studies to demonstrate the proposed approach. Finally, conclusion is made in section 5.

\section{Literature review of the bias correction approach}

Majority of the bias correction approach is based on the Bayesian calibration model proposed by Kennedy and O’Hagan [10] as shown in Eq. (1).

$$
\hat{Y}(\mathbf{x})+\delta(\mathbf{x})=Y-\varepsilon
$$

where $\hat{Y}$ is the baseline model prediction; $\delta$ is the model bias; $Y$ is available test data; $\varepsilon$ is test and measurement error; and $\mathbf{x}$ is a vector of model parameters. The application of this equation is straightforward. For example, maximum deflection of a bridge structure (i.e., $\hat{Y}$ ) under a given loading condition can be estimated by a finite element analysis (FEA) model. Due to model assumptions and simplifications, however, the estimation would not be exactly the same as the real measurement $Y$, if available. With ignorable test and measurement error $\varepsilon$, the difference between the model prediction and real measurement is defined as the model bias $\delta$. If the model significantly underestimates the maximum deflection (i.e., $\delta$ is a large positive value), design error would be created if no tests were run for design verification. Therefore, it is desirable to accurately characterize the model bias as a function of model parameters $\mathbf{x}$ (e.g., parameters that define the topology, shape, and size of the structure) so that the corrected model prediction could be more accurate than the baseline model. In particular, the model parameter $\mathbf{x}$ could take the form of deterministic values, irreducible random parameters (i.e., aleatory uncertainty), and reducible random parameters (i.e., epistemic uncertainty). The Bayesian calibration model addresses the challenge of calibrating the model parameter $\mathbf{x}$, model bias $\delta$, and test and measurement error $\varepsilon$ with limited test data $Y$ at given design configurations. Technically, prior distributions of the distribution parameters (e.g., mean and standard deviation of $\mathbf{x}$, $\delta$, and $\varepsilon$ ) are updated to posterior distributions given available test data $Y$ using a Bayesian updating mechanism.

Model bias should be accurately approximated in the design space if the model would be used to explore new designs without any test data for design verification. Various approaches were proposed for this purpose with the main idea of constructing a meta-model for model bias on the basis of calibrated model bias at available design configurations [4, 11-13]. Among these approaches, regression models are the most popular method because of the well-established research in this field such as the Gaussian process (GP) regression model [11] and the moving least square method [4]. The GP regression model assumes a multivariate normal distribution for the model bias in the design space such that uncertainty of the model bias at each specific design configuration follows a univariate 
normal distribution and their correlations at different design configurations are modeled by an assumed covariance function. The moving least square method directly builds four response surfaces for the first four central moments (i.e., mean, standard deviation, skewness, and kurtosis) of the model bias so that distribution of the model bias can be approximated at any new design configuration using the Pearson system [18]. Due to the curse of dimensionality for regression-based approaches, a Copula-based approach was recently proposed to approximate the model bias through a general statistical relationship between model bias, the baseline model prediction, and the model design variables (i.e., a subset of the model parameter $\mathbf{x}$ that changes when design changes) [12]. However, none of the above approaches considers time dependent model responses where model bias takes the form of a random process at each design configuration instead of a univariate distribution. Hence, it is not feasible to directly apply the bias correction approach for the model with time dependent responses.

\section{Bias correction of time dependent model responses for reliability analysis}

Three research challenges should be addressed in order to apply the bias correction approach for time dependent model responses and reliability analysis. First of all, an accuracy metric needs to be developed to quantify accuracy of the model prediction. Majority of available model accuracy metrics are designed for static (or time independent) model responses such as the U-pooling [14] and the Bayes factor [18]. Though metrics for time dependent responses are also available [19], they are specifically designed for vehicle impact application and uncertainties are not considered in the metric. Secondly, effective approaches need to be developed for time dependent model bias calibration with the aid of the accuracy metric. Finally, effective algorithms should be developed to take account into the time dependent model uncertainty in reliability analysis. Proposed approaches for addressing these challenges are elaborated in the following subsections.

\subsection{Accuracy metric for time dependent responses under uncertainty}

U-pooling metric was proposed by Ferson et al. [14] as an accuracy metric and has been adopted by many researchers in the study of model validation [13, 15-17]. The basic idea is to compare the cumulative distribution function (CDF) difference (i.e., the U-pooling value) between model prediction and test data in the standard Uniform space (or U-space) as shown in Fig. 1. The smaller of area difference indicates the higher of the expected accuracy of the model prediction. For static responses, each test datum $y_{i}$ corresponds to one $u_{i}$ value which is calculated from the CDF value of the model prediction at the same design configuration (i.e., $u_{i}=F_{\hat{M}}\left(y_{i}\right)$ where $F_{\hat{M}}(\square)$ is the CDF of model responses $\hat{M}$ ). To adopt the U-pooling value for time dependent responses, $F_{\hat{\mathbf{M}}}\left(\mathbf{y}_{\mathbf{i}}\right)$ should be calculated where $\mathbf{y}_{\mathbf{i}}$ is the $i^{\text {th }}$ discrete time dependent response from test and $\hat{\mathbf{M}}$ is an arbitrary discrete random process from model prediction. The proposed accuracy metric makes use of the U-pooling approach by addressing two technical components: i) extension of the U-pooling for time dependent responses by extending 1dimensional CDF calculation to $n$-dimensional CDF calculation, and ii) inclusion of the shape difference in the metric. The first component is addressed by discretizing the random process into $n$ time steps such that the calculation of each $u_{i}$ is equivalent to identifying the $n$-dimensional joint CDF value for each discrete random process realization of $\mathbf{y}_{\mathbf{i}}$ (i.e., a vector with $n$ elements). The second component is addressed by quantifying the shape deviation of the model prediction from the test. The proposed accuracy metric for time dependent responses is formulated in Eq. (2).

$$
\Psi\left(\mathbf{Y}^{\mathbf{T}}, \hat{\mathbf{M}}\right)=U\left(u_{1}, \cdots u_{i}, \cdots u_{Q}\right)+\frac{1}{Q} \sum_{i=1}^{Q} S_{i}\left(\mathbf{y}_{\mathbf{i}}^{\mathbf{T}}, \hat{\mathbf{M}}\right) \cong U\left(u_{1}, \cdots u_{i}, \cdots u_{Q}\right)+\frac{1}{Q} \sum_{i=1}^{Q} S_{i}\left(\mathbf{y}_{\mathbf{i}}, \hat{\mathbf{M}}\right)
$$

where $\mathbf{Y}^{\mathbf{T}}$ is the true responses and is represented in a matrix form with multiple test responses or in a vector form if there is only one test response; $\hat{\mathbf{M}}$ indicates model responses (e.g., the baseline model response $\hat{\mathbf{Y}}$ or corrected model responses $\hat{\mathbf{Y}}+\boldsymbol{\delta})$ and is represented by a matrix; $U(\cdot)$ is the U-pooling function; $S_{i}(\cdot, \cdot)$ is the $i^{\text {th }}$ shape deviation function; $\mathbf{y}_{\mathbf{i}}^{\mathbf{T}}$ is the $i^{\text {th }}$ true response represented by a vector; and $Q$ is total number of true or test responses. If test and measurement error $\varepsilon$ is ignorable, then the true responses $\mathbf{Y}^{\mathbf{T}}$ can be approximated by the test responses $\mathbf{Y}$, and $\mathbf{y}_{\mathbf{i}}$ is the $i^{\text {th }}$ test response represented by a vector. Essentially, the smaller value of $\Psi$ indicates the better model accuracy based on available test responses $\mathbf{Y}$. Technical details are elaborated as follows.

\subsection{1 n-dimensional CDF approximation}


Under the assumption of a Gaussian random process, the value of $u_{i}$ can be estimated numerically using a number of algorithms since there is no closed form for $n$-dimensional normal joint CDF [20]. Considering an arbitrary random process, such calculation could be practically difficult. Generally, the sampling approach is able to approximate the $u_{i}$ by counting the number of random process realizations less than or equal to the corresponding realization of $\mathbf{y}_{\mathbf{i}}$ from the test as shown in Eq. (3).

$$
\begin{aligned}
& u_{i}=F_{\hat{\mathbf{M}}}\left(\mathbf{y}_{\mathbf{i}}\right) \cong \sum_{k=1}^{N} S_{k} / N, \\
& \text { where } S_{k}=\left\{\begin{array}{ll}
1 & \hat{m}_{k, j} \leq y_{i, j} \\
0 & \text { elsewhere }
\end{array} \text { for all } j=1, \cdots, n\right.
\end{aligned}
$$

where $F_{\hat{\mathbf{M}}}(\square)$ is the joint CDF of $\hat{\mathbf{M}}$ discretized at $n$ time steps; $N$ is the total number of model response realizations; $y_{i, j}$ indicates the $i^{\text {th }}$ test response value at the $j^{\text {th }}$ discrete time step; and $\hat{m}_{k, j}$ indicates the $k^{\text {th }}$ model response value at the $j^{\text {th }}$ discrete time step. With several test observations of $\mathbf{y}_{\mathbf{i}}(i=1, \ldots, Q)$, corresponding $u_{i}$ can be calculated using Eq. (3) resulting in a U-pooling value as shown in Eq. (2). In particular, the U-pooling value $U \in[0,0.5]$ where 0 and 0.5 indicate the best and worst accuracy, respectively (see Fig.1). It is worth noting that above calculation is general but computationally expensive because each $u_{i}$ estimation requires a magnitude of $N \times n$ comparison operations.

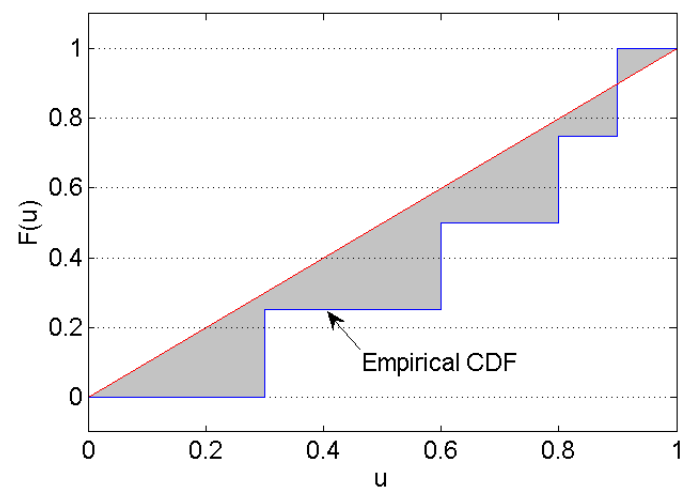

Fig. 1. Illustration of the U-pooling value (i.e., the shaded area)

\subsubsection{Shape deviation}

Unlike static responses, shape difference of time dependent responses between model prediction and test is another important attribute which cannot be simply accounted by the U-pooling value. In other words, models with different accuracy levels could produce the same $u_{i}$ value, resulting in the same U-pooling value in Eq. (2). As shown in Fig. 2a, for example, one test realization $y_{i}$ is located above the prediction bound for both model A and model B, resulting in the same $u_{i}$ value (i.e. $u_{i}=1$ ) for both models according to Eq. (3), although model B is obviously more accurate than model A given the test realization $y_{i}$. It is hence necessary to include the shape deviation in the accuracy metric as defined in the second part of Eq. (2). In particular, the shape deviation for each test realization $\mathbf{y}_{\mathbf{i}}$ is defined as the average absolute response difference between the test and model prediction in a normalized domain as formulated in Eq. (4).

$$
\begin{aligned}
& S_{i}\left(\mathbf{y}_{\mathbf{i}}, \hat{\mathbf{M}}\right)=\frac{1}{N \times n} \sum_{k=1}^{N} \sum_{j=1}^{n}\left|y_{i, j}^{*}-\hat{m}_{k, j}^{*}\right| \\
& \text { where } y_{i, j}^{*}=\left(y_{i, j}-y_{\text {min }}\right) /\left(y_{\text {max }}-y_{\text {min }}\right) ; \hat{m}_{k, j}^{*}=\left(\hat{m}_{k, j}-y_{\text {min }}\right) /\left(y_{\text {max }}-y_{\text {min }}\right) \\
& \text { where } y_{\text {max }}=\max _{k, j}\left\{y_{i, j}, \hat{m}_{k, j}\right\} ; y_{\text {min }}=\min _{k, j}\left\{y_{i, j}, \hat{m}_{k, j}\right\}
\end{aligned}
$$

Fig. $2 \mathrm{~b}$ further illustrates Eq. (4) considering two models provided in Fig. 2a, where each model contains $N$ number of model responses $(k=1,2, \ldots, N)$. It is clearly shown that model B presents smaller shape deviation than model A. In addition, the minimum and maximum shape deviations are 0 and 1 , respectively. 


\subsection{Time dependent model bias calibration and approximation}

This section firstly elaborates the time dependent model bias calibration with the aid of the accuracy metric, then proposes the time dependent model bias approximation at any new design configuration.

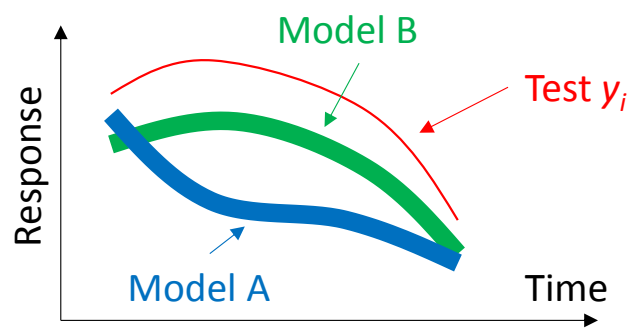

a. Illustration of the same $u_{i}$ value for model $\mathrm{A}$ and model B even though model B is relatively more accurate than model A

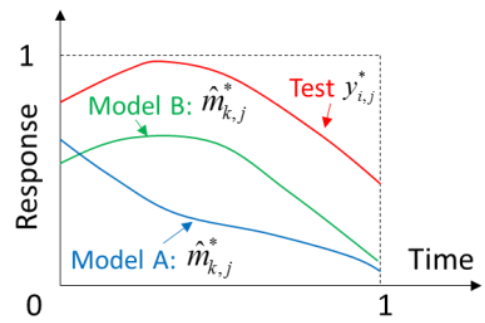

b. Illustration of the shape deviation in a normalized region

Fig. 2. Illustration of the shape deviation in the proposed accuracy metric for time dependent responses

\subsubsection{Time dependent model bias calibration}

The calibration model as shown in Eq. (1) has been used to calibrate the static model bias with well-defined calibration parameters. For example, either the distribution parameters (e.g., the mean and standard deviation) or their hyper-parameters can be treated as calibration parameters with assumed distribution types of the model bias. This calibration model can also be used for time dependent model bias calibration if the calibration parameters can be properly defined.

Considering arbitrary time dependent model bias under uncertainty, the principle component analysis (PCA) is a typical method to characterize the dynamic randomness using only a few uncorrelated random variables and principle components with prescribed accuracy requirement [21]. An arbitrary time dependent model bias in the continuous domain can be formulated in Eq. (5).

$$
\delta(t) \cong \mu(t)+\sum_{k=1}^{R} V_{k} \phi_{k}(t)
$$

where $t$ is the time; $\mu(t)$ is the mean of the time dependent model bias; $\phi_{k}(t)$ is the $k^{\text {th }}$ important principle component; $V_{k}$ is the random variable with zero mean; and $R$ is the number of the vital principle components. It is proposed in this paper that $V_{k}$ is considered as calibration parameters and $\mu(t)$ and $\phi_{k}(t)$ are determined on the basis of the baseline model prediction and corresponding test data. In particular, sufficient model bias realizations can be obtained using the sampling approach such as Monte Carlo simulation (MCS) based on Eq. (1). With discretization of the model bias into $n$ time steps, an $m \times n$ matrix representing the bias can be constructed as

$$
\delta(t) \cong \boldsymbol{\delta}=\left[\begin{array}{cccc}
\delta_{11} & \delta_{12} & \cdots & \delta_{1 n} \\
\delta_{21} & \delta_{22} & \cdots & \delta_{2 n} \\
\vdots & \vdots & \ddots & \vdots \\
\delta_{m 1} & \delta_{m 2} & \cdots & \delta_{m n}
\end{array}\right]
$$

where $\delta_{i j}$ indicates the model bias at the $j^{\text {th }}$ time step for the $i^{\text {th }}$ sampled realization. The mean of the model bias is estimated as

$$
\mu(t) \cong \boldsymbol{\mu}=\left[\bar{\delta}_{\bullet 1}, \bar{\delta}_{\bullet 2}, \cdots, \bar{\delta}_{\bullet n}\right]
$$

where $\bar{\delta}_{. j}$ stands for the average of the $j^{\text {th }}$ time step over the sampled realizations. Hence the variation part of the model bias is expressed as

$$
\mathbf{v}=\left[\begin{array}{cccc}
\delta_{11}-\bar{\delta}_{\bullet 1} & \delta_{12}-\bar{\delta}_{\bullet 2} & \cdots & \delta_{1 n}-\bar{\delta}_{\bullet n} \\
\delta_{21}-\bar{\delta}_{\bullet 1} & \delta_{22}-\bar{\delta}_{\bullet 2} & \cdots & \delta_{2 n}-\bar{\delta}_{\bullet n} \\
\vdots & \vdots & \ddots & \vdots \\
\delta_{m 1}-\bar{\delta}_{\bullet 1} & \delta_{m 2}-\bar{\delta}_{\bullet 2} & \cdots & \delta_{m n}-\bar{\delta}_{\bullet n}
\end{array}\right]
$$

The feature vector $\phi$ can then be obtained by solving an eigen-problem as 


$$
\Sigma \phi=\lambda \phi
$$

where $\lambda$ is the eigenvalue of the covariance matrix $\Sigma(n \times n)$ that is defined as

$$
\Sigma=v^{\mathrm{T}} v
$$

The number of the vital features $(R)$ is dependent on the required accuracy level for preserving the variation part of the matrix $v$, which is determined by the ratio of the first $R$ largest eigenvalues to the summation of all eigenvalues.

Time dependent model bias calibration is thus formulated as

$$
\begin{array}{ll}
\text { Min. } & \Psi\left(\mathbf{Y}^{\mathrm{T}}, \hat{\mathbf{M}}\right) \cong \Psi(\mathbf{Y}, \hat{\mathbf{M}})=\Psi(\mathbf{Y}, \hat{\mathbf{Y}}+\boldsymbol{\delta}) \\
\text { S.T. } & \text { Lower }_{k}^{s} \leq \sigma\left(V_{k}\right) \leq \text { Upper }_{k}^{s} \\
& \text { Lower }_{k}^{\text {skew }} \leq \operatorname{skew}\left(V_{k}\right) \leq \operatorname{Upper}_{k}^{\text {skew }} \\
& \text { Lower }_{k}^{\text {kurto }} \leq \operatorname{kurto}\left(V_{k}\right) \leq \text { Upper }_{k}^{\text {kurto }}
\end{array}
$$

where $\Psi(\cdot, \cdot)$ is the accuracy metric defined in Eq. (2); $\sigma\left(V_{k}\right)$, skew $\left(V_{k}\right)$, and $k u r t o\left(V_{k}\right)$ are standard deviation, skewness, and kurtosis of random variable $V_{k}$, respectively, and they are calibration parameters in the optimization; Lower $_{k}{ }^{x}$ and Upper $_{k}{ }^{x}$ are corresponding lower and upper bounds. The lower bound of standard deviation could be assigned as a small value close to zero such as $1 \mathrm{e}-6$, and the upper bound could be limited to the standard deviation of $\hat{\mathbf{Y}}$. According to the Pearson system [22], a large family of unimodal distributions are covered when absolute skewness ranges from 0 to 2 and kurtosis ranges from 2 to 7 . Hence, the associated bounds are defined as $-2 \leqslant$ $\operatorname{skew}\left(V_{k}\right) \leqslant 2$ and $2 \leqslant \operatorname{kurto}\left(V_{k}\right) \leqslant 7$. These bounds are set for all $V_{k}$ where $k=1,2, \ldots, R$. It is worth noting that if Gaussian random field was assumed for time dependent model bias, only $\sigma\left(V_{k}\right)$ is needed in the above calibration model. This paper employs the Pearson system to approximate the distribution of $V_{k}$ given the first four central moments of $V_{k}$. However, other probability density function (PDF) approximation methods (e.g., Johnson System, maximum entropy principle, saddlepoint approximation, etc.) [23-25] can also be used. The calibrated model bias is finally formulated in Eq. (12) in a discrete manner.

$$
\boldsymbol{\delta}=\boldsymbol{\mu}+\sum_{k=1}^{R} V_{k} \boldsymbol{\phi}_{\mathbf{k}}
$$

where $\mu$ and $\phi_{\mathrm{k}}$ are determined from Eqs. (7) and (9), respectively; $R$ is determined using a 99\% accuracy threshold in the PCA; moments of $V_{k}$ are calibrated through the optimization in Eq. (11); and distributions of $V_{k}$ are constructed using the Pearson system.

\subsubsection{Time dependent model bias approximation}

With successful calibration of time dependent model bias represented in the form of Eq. (12) at several design configurations, regression models of $\mu, \phi_{\mathrm{k}}$ and $V_{k}$ with respect to different design variables $\mathbf{X}$ can be built using various response surface approaches [26] so that model bias at any new design configuration can be approximated. In particular, $\boldsymbol{\mu}$ and $\phi_{\mathrm{k}}$ are deterministic vectors whose regression models can be built at every discrete time step; and $V_{k}$ follows an arbitrary distribution whose statistical moments (i.e., standard deviation, skewness, and kurtosis) can be extracted for building the regression model.

Quality of these regression models plays a critical role for correcting the baseline model prediction with a more credible reliability analysis by incorporating the model uncertainty. Its accuracy mainly depends on three factors including: i) nonlinearity of the model bias in the design space, ii) identified model bias in the design space, and iii) algorithm of the response surface approach. The first factor is determined by accuracy of the baseline model. The second factor is decided by available resources for conducting validation experiments at different design configurations. Intuitively, more validation experiments increase accuracy of the regression models. The third factor is to select a suitable response surface approach. In particular, the Gaussian process (GP) regression model (i.e., the kriging model) was employed in this paper due to its well-known accuracy for many problems. The commonly used isotropic squared exponential covariance function was employed with three hyper-parameters including the lengthscale, signal variance, and noise variance parameters. Those hypermeters were set by optimizing the marginal likelihood [27] to determine the optimal GP regression model based on the training data sets.

\subsection{Reliability analysis considering time dependent model bias}

With approximate model bias at a new design configuration, reliability analysis should be performed for the 
corrected model prediction (i.e., $\hat{\mathbf{Y}}+\boldsymbol{\delta}$ ) instead of the baseline model prediction (i.e., $\hat{\mathbf{Y}}$ ). Essentially, another source of uncertainty (i.e., $\boldsymbol{\delta}$ ) should be included in reliability analysis. Since the model bias $\boldsymbol{\delta}$ is represented by Eq. (12) where $V_{k}$ has the same form as the model random parameter $\mathbf{x}$, any available reliability analysis methods can be used to perform reliability analysis for the corrected model prediction. For example, if MCS were used for reliability analysis, sufficient random samples of $\mathbf{x}$ and $V_{k}$ were firstly generated to simulate the uncertainty of $\hat{\mathbf{Y}}+\boldsymbol{\delta}$, then reliability was calculated from the ratio of safe trials over the total trials. Alternatively, by treating realizations of model bias $\delta$ individually, many reliability values can be computed to form a distribution of reliability due to the uncertainty of model bias. Thus, the former approach obtains the expected reliability considering overall uncertainty from $\delta$, whereas, the alternative approach computes the reliability distribution which might be more useful for safety critical structure design where confidence of the reliability prediction is available due to the uncertainty of the model bias. Other than MCS, many advanced reliability analysis methods, such as the MPP-based approaches [28, 29], the eigenvector dimension reduction methods [30,31], the polynomial chaos expansion methods [32-35], etc., can be employed to further improve the computational efficiency for the reliability analysis.

\section{Case studies}

Two case studies including a thermal problem and a corroded beam problem are employed to demonstrate the proposed accuracy metric, time dependent model bias calibration and approximation, and reliability analysis.

\subsection{Thermal problem}

A thermal problem was developed by Sandia National Laboratory for the study of model validation [36]. The subject is a safety-critical structure, which is schematically shown in Fig. 3. The structure is subject to a constant heat flux rate $q$ with the thickness $L$. Temperature of the structure at location $x$ is expressed in Eq. (13).

$$
T(x, t)=T_{0}+\frac{q L}{k}\left[\frac{(k / \rho) t}{L^{2}}+\frac{1}{3}-\frac{x}{L}+\frac{1}{2}\left(\frac{x}{L}\right)^{2}-\frac{2}{\pi^{2}} \times \sum_{n=1}^{6} \frac{1}{n^{2}} \exp \left(-n^{2} \pi^{2} \frac{(k / \rho) t}{L^{2}}\right) \cos \left(n \pi \frac{x}{L}\right)\right]
$$

where $T_{0}$ is the initial temperature; $k$ is the thermal conductivity; and $\rho$ is the volumetric heat capacity. Specifically, parameters $k$ and $\rho$ have unit-to-unit variability due to the manufacturing tolerance and they were assumed to follow Normal distributions with the mean of 0.0820 and standard deviation of 0.0012 for $k$ and the mean of 400000 and standard deviation of 30113 for $\rho$. For safety reason, probability that surface temperature exceeds a failure temperature, i.e., $T=900^{\circ} \mathrm{C}$, should be less than $1 \%$ after exposure to a heat flux $q=3500 \mathrm{~W} / \mathrm{m}^{2}$ at time $t=1000$ (s). Experiments were conducted at five design configurations as shown in Fig. 4, where four test data sets were available for the first four design configurations and two test data sets were available at the $5^{\text {th }}$ design configuration to verify the model accuracy. Measurement error $\varepsilon$ is ignorable in this problem.

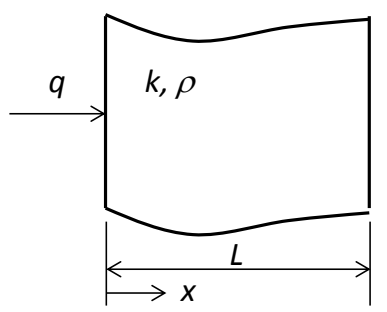

Fig. 3. Schematic view of a safety-critical structure 


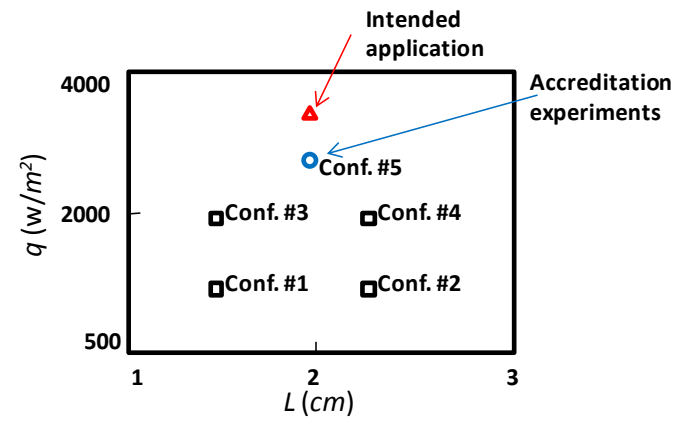

Fig. 4. Validation experiments at five design configurations

\subsubsection{Accuracy metric for the baseline thermal model}

MCS was used for the surface temperature prediction with respect to the time by considering the randomness of material properties. Fig. 5 shows the comparison of temperature responses between four set of test data and the baseline model prediction using MCS with 1000 random samples at four different design configurations. Qualitatively speaking, the baseline model is not accurate and its accuracy varies for different configurations. Table 1 shows the quantitative results of the accuracy metric at four configurations based on Eq. (2). From the U-pooling perspective, the baseline model shows the best accuracy for the $3^{\text {rd }}$ configuration followed by the $1^{\text {st }}$ configuration, and the U-pooling value cannot differentiate the accuracy for the $2^{\text {nd }}$ and $4^{\text {th }}$ configurations. According to the average shape deviation $\bar{S}$, the $3^{\text {rd }}$ configuration again presents the best accuracy followed by the $1^{\text {st }}, 2^{\text {nd }}$, and $4^{\text {th }}$ configurations. Overall, the baseline model presents the best and worst accuracy for the $3^{\text {rd }}$ and $4^{\text {th }}$ configurations, respectively.

Table 1. Accuracy metric of the baseline model prediction at four design configurations

\begin{tabular}{llll}
\hline \hline & $U$ & $\bar{S}$ & $\Psi$ \\
\hline Conf. \#1 & 0.2999 & 0.1116 & 0.4116 \\
Conf. \#2 & 0.3956 & 0.1295 & 0.5251 \\
Conf. \#3 & 0.2362 & 0.0779 & 0.3142 \\
Conf. \#4 & 0.3957 & 0.1422 & 0.5380 \\
\hline \hline
\end{tabular}

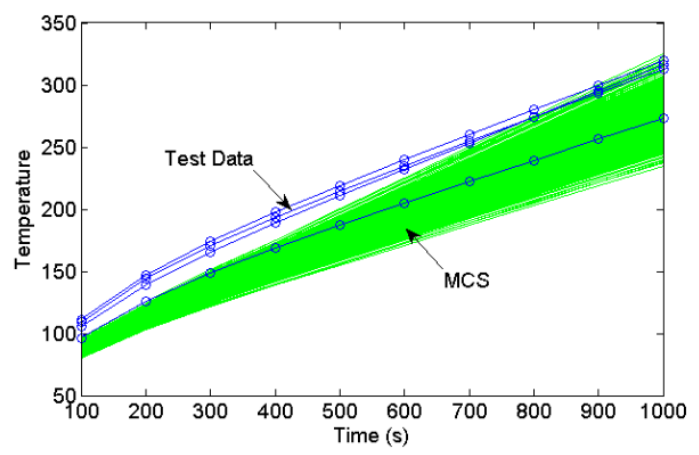

a. Conf. \#1

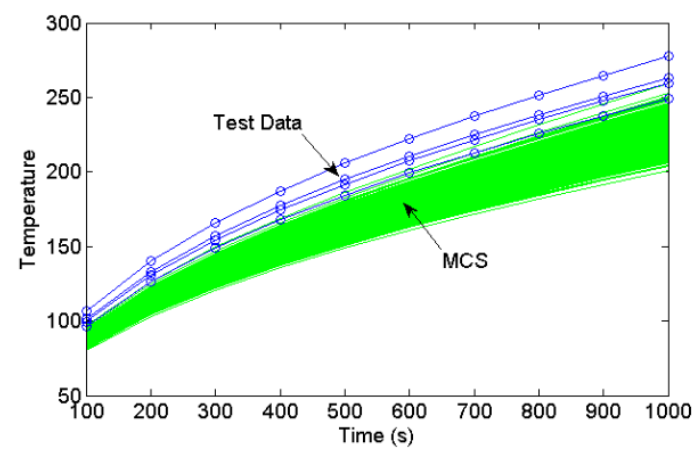

b. Conf. \#2 

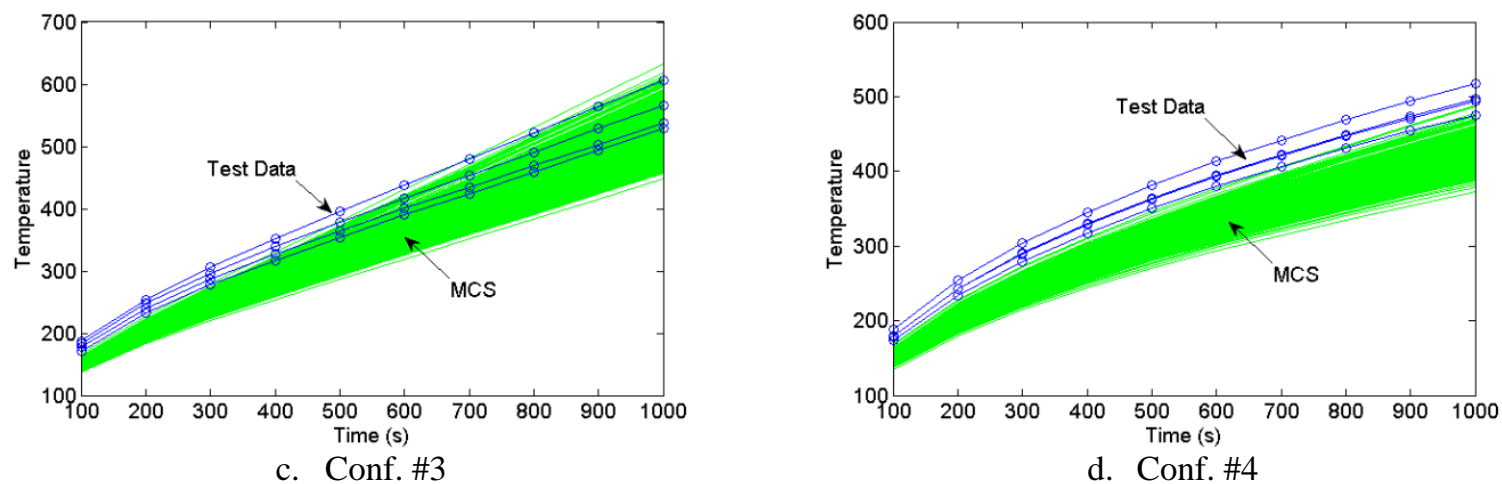

Fig. 5. Comparison of temperature responses between the baseline model and four set of test data at four different design configurations

\subsubsection{Model bias calibration and approximation for the baseline thermal model}

The proposed calibration model in Eq. (11) was employed to calibrate the time dependent model bias at four training design configurations. With predetermined mean $\mu$ and features $\phi_{\mathrm{k}}$ of the time dependent model bias, statistical moments of $V_{k}$ were calibrated to minimize the accuracy metric $\Psi$ so that the agreement between the corrected model prediction and test data was maximized. The calibration history of $\Psi$ is listed in Table 2 at four training design configurations. Obviously, accuracy of the corrected model prediction was significantly improved as compared to the accuracy metric in Table 1. Fig. 6 further shows the comparison of temperature responses between the corrected model prediction and the test data.

Table 2. Optimization history of the accuracy metric $\Psi$ for the thermal problem

\begin{tabular}{lllll}
\hline \hline Iteration & Conf. \#1 & Conf. \#2 & Conf. \#3 & Conf. \#4 \\
\hline 0 & 0.099 & 0.088 & 0.098 & 0.103 \\
1 & 0.085 & 0.086 & 0.092 & 0.102 \\
2 & 0.070 & 0.039 & 0.050 & 0.037 \\
3 & 0.069 & 0.034 & 0.043 & 0.034 \\
4 & 0.069 & 0.033 & 0.042 & 0.034 \\
Optimal & $\mathbf{0 . 0 6 9}$ & $\mathbf{0 . 0 3 3}$ & $\mathbf{0 . 0 4 2}$ & $\mathbf{0 . 0 3 4}$ \\
\hline \hline
\end{tabular}

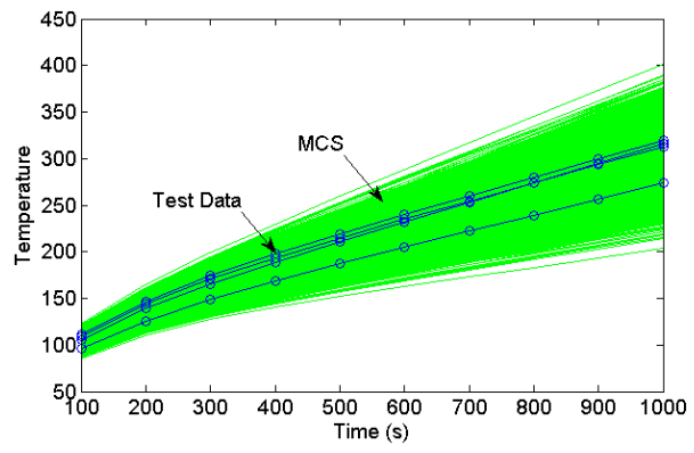

a. Conf. \#1

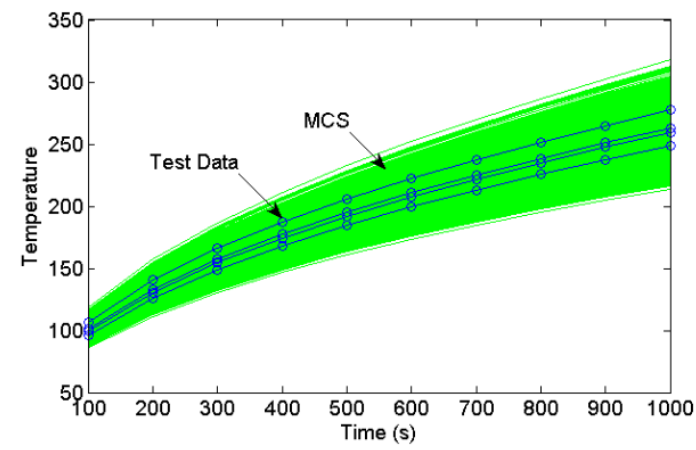

b. Conf. \#2 


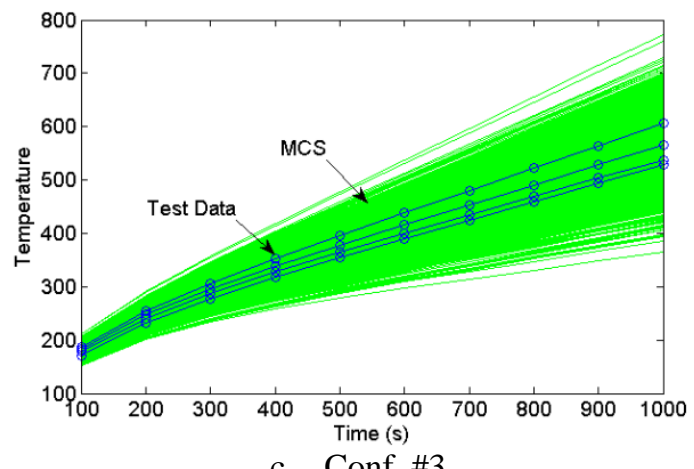

c. Conf. \#3

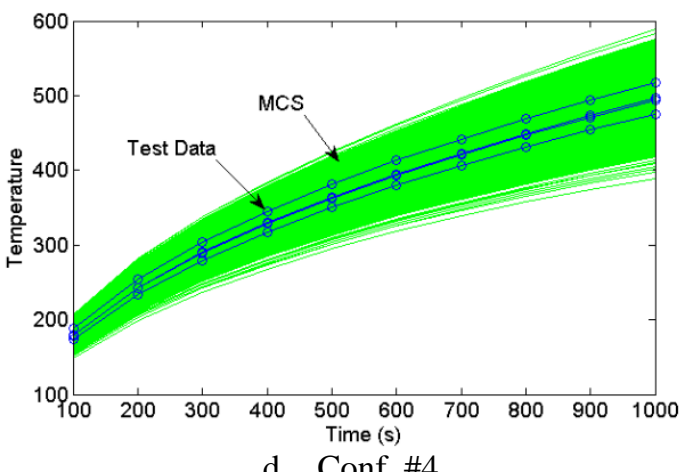

d. Conf. \#4

Fig. 6. Comparison of temperature responses between the corrected model and four set of test data at four different design configurations

With successful calibration of the time dependent model bias at four training design configurations, response surfaces of $\mu, \phi_{\mathrm{k}}$, and statistical moments of $V_{k}$ were constructed as functions of the heat flux rate $q$ and structure thickness $L$ using the GP regression model. The approximate model bias at the $5^{\text {th }}$ configuration is shown in Fig. 7 where 1000 random samples of the bias were generated using MCS based on Eq. (12) and the centered solid line is the mean of the bias. To demonstrate the effectiveness of the proposed bias approximation, the bias was employed to correct the baseline model prediction at the $5^{\text {th }}$ configuration. In Fig. 8, two set of accreditation experiments were used to verify the accuracy improvement of the baseline model after incorporating the approximate model bias for the design configuration \#5. Quantitatively, the value of $\Psi$ was reduced $47 \%$ indicating significant accuracy improvement of the baseline model after adding the approximate model bias as shown in Table 3 .

Table 3. Accuracy metric for Conf. \#5

\begin{tabular}{llll}
\hline \hline & $U$ & $\bar{S}$ & $\Psi$ \\
\hline Conf. \#5: Baseline model & 0.2950 & 0.0752 & 0.3702 \\
Conf. \#5: Corrected model & 0.1836 & 0.0122 & 0.1959 \\
\hline \hline
\end{tabular}

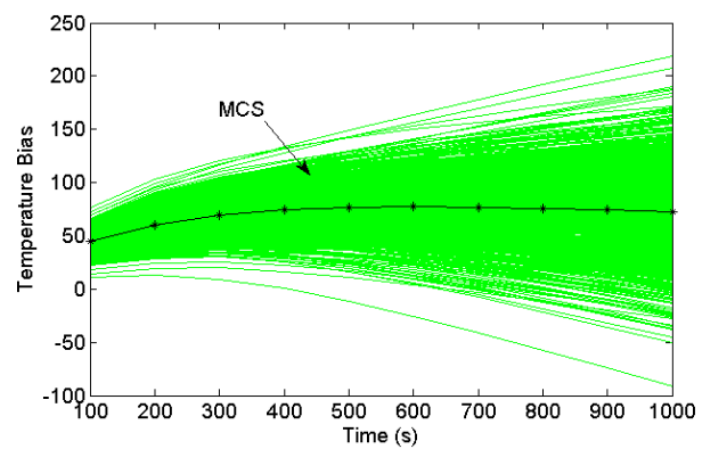

Fig. 7. Model bias of the temperature responses approximated at new design configuration \#5 for the baseline model 


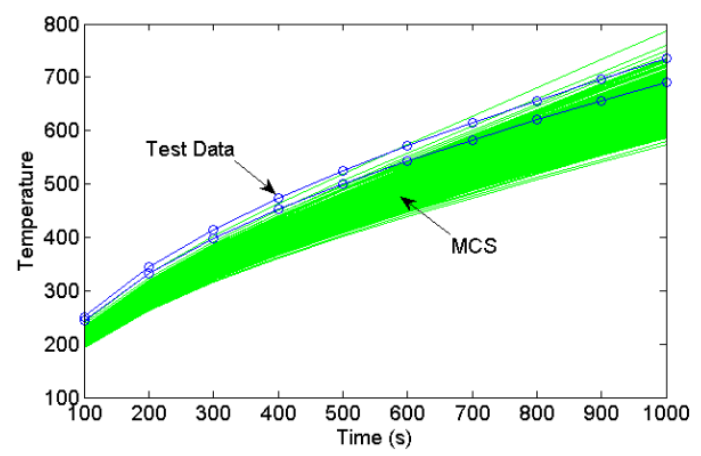

a. Baseline model vs. test data

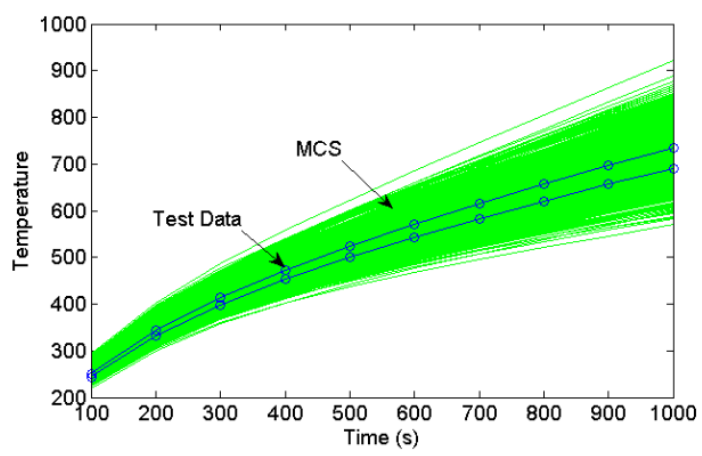

b. Corrected baseline model vs. test data

Fig. 8. Comparison of temperature responses between models and two set of test data at Conf. \#5

\subsubsection{Reliability analysis at the intended operation condition}

For safety concern, reliability of the structure should be at least $99 \%$ at the intended operation condition (i.e., heat flux rate $q=3500 \mathrm{~W} / \mathrm{m}^{2}$ ) where experiments are not available or too expensive to be conducted for reliability analysis. The baseline model in Eq. (13) thus was employed for reliability analysis where the reliability was computed as $99.9 \%$ using the MCS with 100,000 samples. Therefore, the reliability constraint is met according to the baseline model. However, significant model bias was observed for this baseline model at several training design configurations, which indicates that the estimated reliability from the baseline model could be wrong and misleading. The reliability, therefore, has to be reevaluated by considering the potential model bias at the intended operation condition. Similar to the bias estimation for conf. \#5, the bias at the intended operation condition was approximated and added to the baseline model for reliability analysis resulting in an expected reliability value of $84.1 \%$ as shown in Fig. 9. Furthermore, a reliability distribution was obtained as shown in Fig. 9 by treating 1,000 realizations of model bias individually. This distribution is useful to draw confidence interval of the reliability prediction due to the model uncertainty. Results of this case study clearly demonstrate that model uncertainty plays a significant role in reliability analysis.

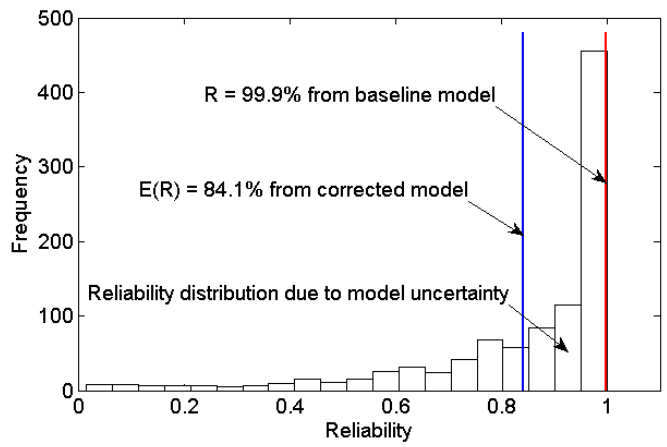

Fig. 9. Comparison of reliability prediction between the baseline model and corrected model with model uncertainty 4.2 A corroded beam problem

A corroded beam problem $[37,38]$ was modified to further demonstrate the proposed approach. As shown in Fig. 10 , the cross section of the beam is rectangular with initial width of $a_{0}$ and height of $b_{0}$. Both the width and height decrease at a rate of $r_{1 i}$ and $r_{2 i}$ at the $i^{\text {th }}$ year respectively due to the corrosion. The corrosion rate is assumed to be uniform along the length of the beam. The beam is subject to its own weight and a random load $F$ at the center of the beam. The failure occurs when the external stress exceeds the strength of the beam which decays over the time due to the corrosion. The true limit sate function is formulated in Eq. (14) with the assumed true decay rate of $r_{1 i}$ and $r_{2 i}$.

$$
g=F L / 4+\rho a_{0} b_{0} L^{2} / 8-\left(a_{0}-2 \sum_{i} r_{1 i}\right)\left(b_{0}-2 \sum_{i} r_{2 i}\right) \sigma_{u} / 4
$$

where $\sigma_{u}$ is the ultimate strength; $\rho$ is the density; $L$ is the length of the beam; and $r_{1 i}=1.1 r_{0 i}$ and $r_{2 i}=1.5 r_{0 i}$, where $r_{0 i}$ is a nominal decay rate at the $i^{\text {th }}$ year. It is assumed in this example that the true decay rate of $r_{1 i}$ and $r_{2 i}$ cannot be directly measured for an intended operation condition with a relatively large beam size defined by $a_{0}$ and $b_{0}$ due to limited experiment resources. Hence, the baseline model employs a nominal decay rate $r_{0 i}$ on the basis of historical 
data. Thus, the baseline model of the limit state function which is employed for reliability prediction is formulated as

$$
g=F L / 4+\rho a_{0} b_{0} L^{2} / 8-\left(a_{0}-2 \sum_{i} r_{0 i}\right)\left(b_{0}-2 \sum_{i} r_{0 i}\right) \sigma_{u} / 4
$$

In short, Eq. (14) is the true model assumed to be unknown and is only used for reference, and Eq. (15) is used as a baseline model for reliability analysis for a time period of 30 years (i.e., $i=1,2, \ldots, 30$ ). At the intended operation condition, model parameters and their properties are listed in Table 4, where uncertainties are considered for $a_{0}, b_{0}$ and $\sigma_{u}$ because of the manufacturing tolerance. The loading condition $F$ is assumed to follow a Normal distribution for simplicity. The nominal decay rate $\mathrm{r}_{0 i}$ is provided in Table 5 where the decay rate increases over the time to simulate the aging effect.

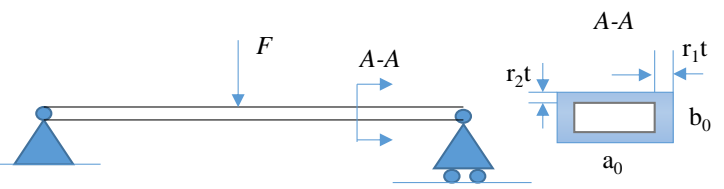

Fig. 10. Schematic view of a corroded beam problem

Table 4. Model parameters and properties of a corroded beam problem at the intended operation condition

\begin{tabular}{llll}
\hline \hline Variable & Mean & Standard deviation & Distribution \\
\hline$a_{0}$ & $0.2 \mathrm{~m}$ & $0.01 \mathrm{~m}$ & Normal \\
$b_{0}$ & $0.04 \mathrm{~m}$ & $0.004 \mathrm{~m}$ & Normal \\
$\sigma_{u}$ & $2.4 \mathrm{e} 8 \mathrm{~Pa}$ & $2.4 \mathrm{e} 7 \mathrm{~Pa}$ & Lognormal \\
$F$ & $200,000 \mathrm{~N}$ & $40,000 \mathrm{~N}$ & Normal \\
$L$ & $5 \mathrm{~m}$ & 0 & Deterministic \\
$\rho$ & $78.5 \mathrm{kN} / \mathrm{m}^{3}$ & 0 & Deterministic \\
\hline \hline
\end{tabular}

Table 5. Nominal decay rate over 30 years

\begin{tabular}{|c|c|c|c|c|c|c|c|c|c|c|}
\hline . & 1 & 2 & 3 & I & 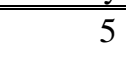 & 0 & r & 8 & 9 & $\begin{array}{r}10 \\
8 \text { - } 5\end{array}$ \\
\hline$r_{0 i}$ & $5 e-5$ & $2 e-5$ & $5.4 \mathrm{e}-5$ & $5.6 e-5$ & $5.8 \mathrm{e}-5$ & $6 e-5$ & $.2 \mathrm{e}-5$ & $4 \mathrm{e}-5$ & $6 e-5$ & $.8 \mathrm{e}-5$ \\
\hline & 11 & 11 & 13 & 14 & 15 & 16 & 17 & 18 & 19 & 20 \\
\hline & -5 & e- -5 & $8 e-5$ & $2 e-5$ & e- -5 & $e-5$ & $4 e-5$ & $e-5$ & $2 e-4$ & $6 e-4$ \\
\hline & 2 & 22 & 23 & 24 & 2. & 26 & 27 & 28 & 29 & 30 \\
\hline$r_{0 i}(\mathbf{m} / \mathbf{y r})$ & $10 \mathrm{e}-4$ & $.16 \mathrm{e}-4$ & $.22 \mathrm{e}-4$ & $1.28 \mathrm{e}-4$ & $1.34 \mathrm{e}-4$ & $1.40 \mathrm{e}-4$ & $1.46 \mathrm{e}-4$ & $1.52 \mathrm{e}-4$ & $1.58 \mathrm{e}-4$ & $1.64 \mathrm{e}-4$ \\
\hline
\end{tabular}

It is assumed that the true decay rate cannot be directly measured at the intended operation condition with relatively large size of $a_{0}$ and $b_{0}$. Model bias of the baseline model needs to be firstly calibrated at other operation conditions (e.g., downsized sample tests for $a_{0}$ and $b_{0}$ ) and then approximated at the intended operation condition. In this example, only one set of virtual test data of the beam strength was employed at four training design configurations where the means of $a_{0}$ and $b_{0}$ were downsized as shown in Table 6 . The virtual test data of the beam strength were obtained from Eq. (16) where only one random realization was extracted using MCS considering the randomness of $a_{0}, b_{0}$, and $\sigma_{u}$ at each configuration. For practical concerns, the virtual test could be potentially replaced by real experiments with the aid of the highly accelerated life testing [39-41]. Fig. 11a shows such an example of the comparison between the baseline model prediction and one virtual test data for the $1^{\text {st }}$ configuration. With successful calibration of the time dependent model bias, the corrected model prediction shows much higher degree of agreement as shown in Fig. 11b. Similar work was conducted for other three training configurations and finally response surfaces of the model bias were built as functions of $a_{0}$ and $b_{0}$.

$$
\text { Strength }_{i}=\left(a_{0}-2 \sum_{i} r_{1 i}\right)\left(b_{0}-2 \sum_{i} r_{2 i}\right) \sigma_{u} / 4
$$

Table 6. Four training configurations

\begin{tabular}{lllll}
\hline \hline Configuration & Variable & Mean & STD & Distribution \\
\hline Conf. \#1 & $a_{0}$ & $0.1 \mathrm{~m}$ & $0.01 \mathrm{~m}$ & Normal \\
& $b_{0}$ & $0.02 \mathrm{~m}$ & $0.004 \mathrm{~m}$ & Normal \\
Conf. \#2 & $a_{0}$ & $0.15 \mathrm{~m}$ & $0.01 \mathrm{~m}$ & Normal \\
& $b_{0}$ & $0.02 \mathrm{~m}$ & $0.004 \mathrm{~m}$ & Normal \\
\hline \hline
\end{tabular}




\begin{tabular}{lllll}
\hline \hline Conf. \#3 & $a_{0}$ & $0.1 \mathrm{~m}$ & $0.01 \mathrm{~m}$ & Normal \\
& $b_{0}$ & $0.03 \mathrm{~m}$ & $0.004 \mathrm{~m}$ & Normal \\
Conf. \#4 & $a_{0}$ & $0.15 \mathrm{~m}$ & $0.01 \mathrm{~m}$ & Normal \\
& $b_{0}$ & $0.03 \mathrm{~m}$ & $0.004 \mathrm{~m}$ & Normal \\
\hline \hline
\end{tabular}

Reliability Analyses were finally conducted at the intended operation condition for the baseline model, the corrected baseline model considering approximated model bias, and the reference model over a period of 30 years and results are shown in Fig. 12. Obviously, the baseline model significantly underestimates the probability of failure. The corrected model accurately predicts the reliability with the aid of the identified model bias at the intended operation configuration. Furthermore, 95\% confidence intervals (CIs) were provided considering the uncertainty from the model bias.

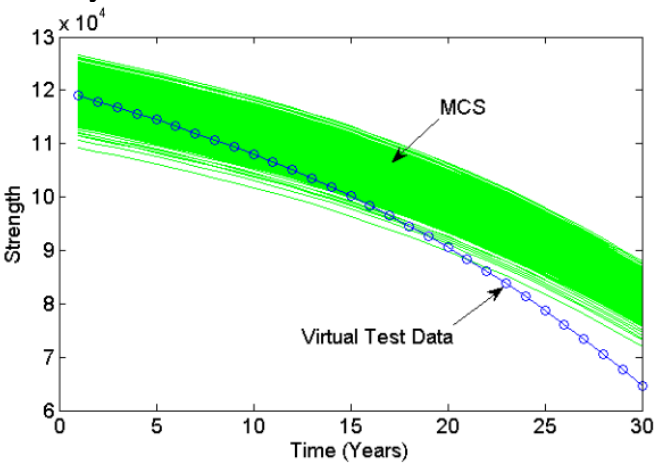

a. Before bias calibration

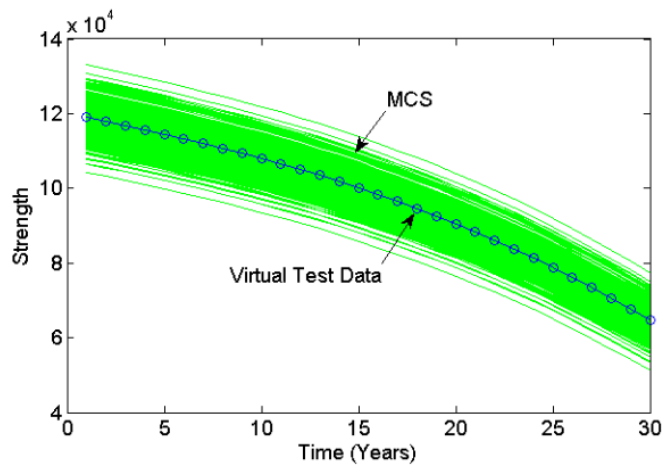

b. After bias calibration

Fig. 11. Comparison of the beam strength responses between model predictions and one virtual test data at the $1^{\text {st }}$ training configuration

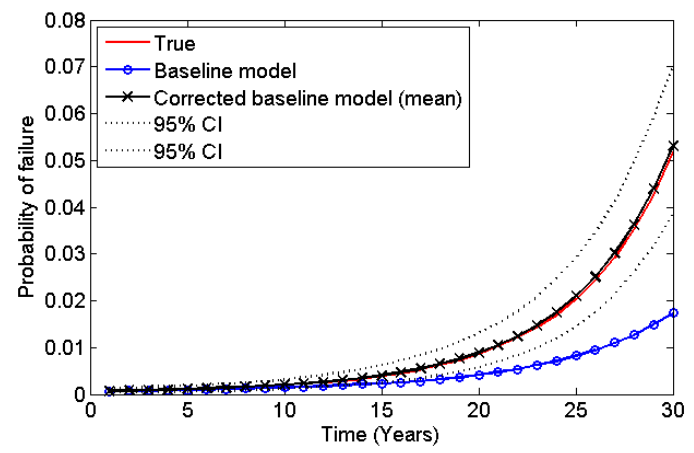

Fig. 12. Reliability estimation of a corroded beam over 30 years

\section{Conclusions}

This paper proposed a time dependent model bias correction approach for model based reliability analysis. Contribution of this paper is three-fold. First of all, a new accuracy metric for time dependent model responses was proposed which is composed of the U-pooling and shape deviation. The U-pooling verifies the model accuracy from a distribution matching point of view and the shape deviation determines the model accuracy from a time dependent response matching viewpoint. Therefore, the integration of them nicely meets the need of an accuracy metric for time dependent responses under uncertainty. The second contribution is to propose a calibration model for time dependent model bias using the PCA so that only limited number of calibration parameters are needed and the calibration can be effectively conducted similar to the static model bias calibration. The third contribution is to propose the time dependent model bias approximation through building the response surfaces of PCA model components (i.e., $\mathbf{u}, \phi_{\mathbf{k}}$, and $V_{k}$ ) so that the approximate model bias keeps the same form as a PCA model which makes it possible to seamlessly integrate the time dependent model bias with the baseline model for reliability analysis. The effectiveness of the proposed approach was demonstrated by two case studies with time dependent model responses. One important finding is that reliability analysis on the basis of a low fidelity baseline model could be wrong and misleading and the proposed approach can effectively increase the accuracy of the baseline model for 
reliability analysis.

\section{Acknowledgments}

The research was supported by Ford Motor Company and partially supported by National Science Foundation under the Award Number 1507198.

\section{References}

[1] Hill RG, Trucano TG. Statistical validation of engineering and scientific models: Background. Sandia National Laboratories, 1999, SAND99-1256.

[2] Thacker BH, Doebling SW, Hemez FM, Anderson MC, Pepin JE, Rodriguez EA. Concepts of model verification and validation. Los Alamos National Lab., Los Alamos, 2004, LA-14167.

[3] Babuska I, Oden JT. Verification and validation in computational engineering and science: Basic concepts. Comput. Methods Appl. Mech. Engrg. 2004; 193: 4057-4066.

[4] Xi Z, Fu Y, Yang R-J. Model bias characterization in the design space under uncertainty. Int. J. Perform. Engrg. 2013; 9 (4): 433-444.

[5] Zhan Z, Fu Y, Yang R-J. On stochastic model interpolation and extrapolation methods for vehicle design. SAE Int. J. Mater. Manf. 2013; 6(3).

[6] Zhan Z, Fu Y, Yang R-J, Xi Z, Shi L. A Bayesian inference based model interpolation and extrapolation. SAE Int. J. Mater. Manf. 2012; 5(2): 357-364.

[7] Jiang Z, Chen W, Fu Y, Yang R-J. Reliability-based design optimization with model bias and data uncertainty SAE Int. J. Mater. Manf. 2013; 6(3).

[8] Xi Z, Fu Y, Yang R-J. An ensemble approach for model bias prediction. SAE Int. J. Mater. Manf. 2013; 6(3)

[9] Higdon D, Gattiker J, Williams B, Rightley M. Computer model calibration using high-dimensional output. J. Amer. Statist. Assoc. 2008; 103(482).

[10] Kennedy MC, O’Hagan A. Bayesian calibration of computer models. J. Roy. Statist. Soc., Ser. B. 2001; 63: 425-464.

[11] Arendt PD, Chen W, Apley DW. Updating predictive models: calibration, bias correction and identifiability. Proc. ASME Des. Engrg. Tech. Conf. 2010; 1089-1098.

[12] Xi Z, Pan H, Fu Y, Yang R-J. A Copula-based approach for model bias characterization. SAE Int. J. Passeng. Cars - Mech. Syst. 2014; 7 (2):781-786.

[13] Xiong, Y., Chen, W., Tsui, K.-L., Apley, D.W., A better understanding of model updating strategies in validating engineering models, (2009) Computer Methods in Applied Mechanics and Engineering, 198 (1516), pp. 1327-1337.

[14] Ferson S, Oberkampf WL, Ginzburg L. Model validation and predictive capability for the thermal challenge problem. Comput. Methods in Appl. Mech. Eng. 2008; 197: 2408-30.

[15] Liu, Y., Huang, H.-Z., Zhang, X., A data-driven approach to selecting imperfect maintenance models, (2012) IEEE Transactions on Reliability, 61 (1), art. no. 6045309, pp. 101-112.

[16] Li, W., Chen, W., Jiang, Z., Lu, Z., Liu, Y., New validation metrics for models with multiple correlated responses, (2014) Reliability Engineering and System Safety, 127, pp. 1-11.

[17] Jung, B.C., Park, J., Oh, H., Kim, J., Youn, B.D., A framework of model validation and virtual product qualification with limited experimental data based on statistical inference, (2015) Structural and Multidisciplinary Optimization, 51 (3), pp. 573-583.

[18] Ling,Y, Mahadevan S, Quantitative model validation techniques: New insights. Reliab. Engrg. Syst. Saf. 2013; 111: 217-231.

[19] Sarin H, Kokkolaras M, Hulbert G, Papalambros P, Barbat S, Yang R-J. Comparing time histories for validation of simulation models: Error measures and metrics. J. Dyn. Syst. Meas. Control. Trans. ASME. 2010; 132 (6), 061401.

[20] Genz A. Computation of multivariate normal and t probabilities. Springer. ISBN 978-3-642-01689-9, 2009.

[21] Xi Z, Youn BD, Hu C. Random field characterization considering statistical dependence for probability analysis and design. J. Mech. Des. 2010; 132, 101008.

[22] Pearson K. Mathematical contributions to the theory of evolution, X: supplement to a memoir on skew variation. Philos. Trans. R. Soc. Lond. A. 1901; 197: 443-459.

[23] Daniels HE. Saddlepoint approximations in statistics. Ann. Math. Stat. 1954; 25(4): 631-650.

[24] Jaynes ET. Information theory and statistical mechanics. Phys. Rev. 1957; 620-630. 
[25] Johnson NL, Kotz S, Balakrishnan N. Continuous univariate distributions. Wiley, New York, 1994.

[26] Myers RH, Montgomery DC. Response surface methodology: Process and product in optimization using designed experiments. Wiley \& Sons, 1995; New York, NY, USA.

[27] Rasmussen, C.E., and Williams, C.K.I., Gaussian Processes for Machine Learning, The MIT Press, 2006, ISBN $0-262-18253-X$.

[28] Hasofer AM, Lind NC. Exact and invariant second-moment code format. ASCE J. Engrg. Mech. 1974; 100, No. 1, 111-121.

[29] Tvedt L. Two second-order approximations to the failure probability. Section on Structural Reliability, A/S Vertas Research, Hovik, Norway. 1984.

[30] Xu H, Rahman S. A generalized dimension-reduction method for multidimensional integration in stochastic mechanics. Int. J. Numer. Method. Engrg. 2004; 61: 1992-2019.

[31] Youn BD, Xi Z, Wang P. Eigenvector dimension-reduction (EDR) method for sensitivity-free probability analysis. Struct. Multidiscip. Optim. 2008; 37, No. 1, 13-28.

[32] Hu C, Youn BD. Adaptive-sparse polynomial chaos expansion for reliability analysis and design of complex engineering systems. Struct. Multidiscip. Optim. 2011; 43(3): 419-442.

[33] Blatman G, Sudret B. An adaptive algorithm to build up sparse polynomial chaos expansions for stochastic finite element analysis. Prob. Engrg. Mech. 2010; 25(2): 183-197.

[34] Oladyshkin S, Nowak W. Data-driven uncertainty quantification using the arbitrary polynomial chaos expansion. Reliab. Engrg. Syst. Saf. 2012; 106: 179-190.

[35] Coelho RF, Lebron J, Bouillard P. Hierarchical stochastic metamodels based on moving least squares and polynomial chaos expansion: Application to the multiobjective reliability-based optimization of space truss structures, Struct. Multidiscip. Optim. 2011; 43(5): 707-729.

[36] Dowding KJ, Pilch M, Hills RG. Formulation of the thermal problem. Comput. Methods Appl. Mech. Engrg. 2008; 197: 2385-2389.

[37] Andrieu-Renaud C, Sudret B, Lemaire M. The PHI2 method: A way to compute time-variant reliability. Reliab. Engrg. Syst. Saf. 2004; 84(1): 75-86.

[38] Hu Z, Du X. Time-dependent reliability analysis with joint upcrossing rates. Struct. Multidiscip. Optim. 2013; 48(5): 893-907.

[39] Torres-Acosta, A.A., Navarro-Gutierrez, S., Terán-Guillén, J., Residual flexure capacity of corroded reinforced concrete beams, (2007) Engineering Structures, 29 (6), pp. 1145-1152.

[40] Apostolopoulos, C.A., Papadakis, V.G., Consequences of steel corrosion on the ductility properties of reinforcement bar, (2008) Construction and Building Materials, 22 (12), pp. 2316-2324.

[41] Sahmaran, M., Li, V.C., Andrade, C., Corrosion resistance performance of steel-reinforced engineered cementitious composite beams, (2008) ACI Materials Journal, 105 (3), pp. 243-250. 